\title{
A Novel Smart Resistive-Capacitive Position Sensor
}

\author{
Xiujun Li and Gerard C. M. Meijer
}

\begin{abstract}
A novel smart resistive-capacitive angular position sensor is presented. The main advantages of this low-cost system are its simplicity, high stability and high reliability. A very linear oscillator is used in the processing circuit to convert the position quantity to a period-modulated signal which can directly be read out by a microcontroller. The system does not need an A/D converter. The nonlinearity of the smart angular position sensor system is less than $\pm 0.3 \%\left( \pm 0.9^{\circ}\right)$ over the range of $270^{\circ}$.
\end{abstract}

\section{INTRODUCTION}

C ONSISTING of a single wire with a sliding contact, the resistive potentiometer is one of the simplest and most efficient position sensors. Its disadvantages are low accuracy and linearity. Moreover, the long-term stability of this system is bad since the sliding contact is directly in contact with the resistive element of the potentiometer. The position-sensitive detector (PSD) which is an optical potentiometer [1]-[3] is an interesting alternative which is suited to be implemented as an integrated circuit. But this system can only measure displacement over a small range $(3 \mathrm{~mm})$, and the cost and energy consumption of the PSD and the LED are rather high. These drawbacks are overcome in the novel resistivecapacitive potentiometer which is described in this paper. This potentiometer is used as a sensing element for a smart resistive-capacitive position sensor.

The smart resistive-capacitive position sensor is suited to replace both linear and circular potentiometers. In this paper, we limit ourselves to the discussion of an angular encoder for the measurement range of $270^{\circ}$.

\section{BASIC PRINCIPLE}

Fig. 1 shows the entire resistive-capacitive position sensor system. The resistive-capacitive sensing element is a modified potentiometer with a sliding contact which is not directly in contact with the resistive layer of the potentiometer. The slide forms an electrode which is capacitively coupled to the resistive layer. The advantages are low cost, high long-term stability and a wide measuring range.

The 3-signal approach presented in [1] and [5] is used to obtain a simple and accurate signal processor. In this processor, an oscillator is used of which the period of oscillation is linearly related to the position. This period-modulated signal can directly be read out by a misrocontroller. There is no need for an additional A/D converter or for other analog processing circuits.

The resistive-capacitive sensing element consists mainly of two parts: a rotating electrode and a resistive layer. A simple

Manuscript received May 10, 1994; revised January 31, 1995.

The authors are with the Department of Electrical Engineering, Delft University of Technology, 2628 CD Delft, The Netherlands.

IEEE Log Number 9411490.

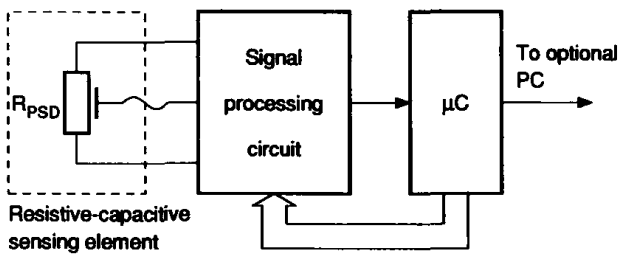

Fig. 1. An overview of the smart resistive-capacitive angular position sensor for a linear potentiometer.

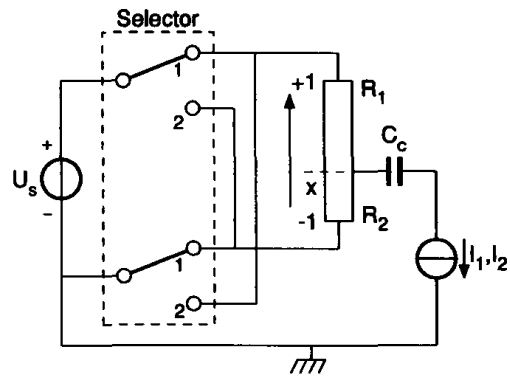

Fig. 2. A simple equivalent model of the resistive-capacitive sensing element.

equivalent model of the resistive-capacitive position sensor is shown in Fig. 2.

The capacitor $C_{c}$ represents the equivalent capacitance between the sliding electrode and the resistive layer. The sum of the resistances $R_{1}$ and $R_{2}$ is the resistance $R_{\mathrm{PSD}}$ of the resistive layer. When $I_{1}$ and $I_{2}$ represent the currents through $C_{c}$ in the position " 1 " and " 2 " of the switches respectively and we denote the relative position by a number $x$, which varies from -1 for the bottom position to +1 for the top position, then we can find that

$$
x=\frac{R_{2}-R_{1}}{R_{1}+R_{2}}=\frac{I_{1}-I_{2}}{I_{1}+I_{2}} .
$$

The position $x$ is only the function of the resistances $R_{1}$ and $R_{2}$ and is not related to capacitance $C_{c}$. This is an important property because it means that the measurement is immune to the electrode distance and the mechanical tolerances which result from deviations or nonuniformity of the electrode distance.

\section{Signal Processing}

According to the 3-signal method presented in [1], [5], we measure successively a variable quantity $M_{x}$ and two reference quantities $M_{1}$ and $M_{2}$ in an identical way, using the same system. The measuring result is the ratio

$$
M=\left(M_{x}-M_{1}\right) /\left(M_{2}-M_{1}\right) .
$$


When the system is linear, then in this ratio the influence of the unknown offset and the unknown gain of the measurement system is eliminated.

In order to obtain the position $x$, the three currents $I_{1}, I_{2}$, and $I_{0}$ have to be measured. The current $I_{0}$ is permitted to be zero. The three currents can be converted into periodmodulated signals, using a capacitance-controlled oscillator which generates square-wave output signals where periods $T_{p i}$ are related to the current by the linear equation

$$
T_{p i}=a I_{i}+b \quad(i=0,1,2) .
$$

When $I_{0}=0$, we find

$$
\frac{T_{p 1}-T_{p 2}}{T_{p 1}+T_{p 2}-T_{p 0}}=\frac{I_{1}-I_{2}}{I_{1}+I_{2}}=x .
$$

Fig. 3 shows the electronic circuitry for the smart resistivecapacitive position sensor system.

The modified Martin oscillator [4], [5] is composed of an operational amplifier $A_{1}$, a comparator $\mathrm{CP}$, an inverter, the capacitances $C_{1}, C_{2}$ and a resistance $R_{0}$. The microcontroller can directly read out the period-modulated signals from $V_{4}$ and control the selector (two NAND gates). A buffer is used between the oscillator and the selector to eliminate undesired interactions.

\section{THE INFLUENCE OF SYSTEMATIC ERRORS}

In [1] it is shown that the system is immune to most of the nonidealities of the opamp and the comparator, such as slewing, limitations of bandwidth and gain, offset voltages and input bias currents. These nonidealities only cause additive or multiplicative errors which are eliminated by the 3-signal method. But some effects cannot be eliminated by the 3-signal method in this system, as will be discussed now:

\section{A. The Effect of Two NAND Gates Which Have Different $O N$ Resistances $R_{\mathrm{ON}}$}

The output stages of two NAND gates shown in Fig. 3 will cause influence on the measured position. It is assumed the resistances $R_{\mathrm{PON} 1}, R_{\mathrm{NON} 1}$ and $R_{\mathrm{PON} 2}, R_{\mathrm{NON} 2}$ represent respectively the ON resistances of the output stages of two NAND gates under the high and low output levels. A straightforward calculation of the offset of $R_{\mathrm{ON}}$ for the measured position can be approximated by:

$$
\begin{aligned}
\frac{T_{p 1}-T_{p 2}}{T_{p 1}+T_{p 2}-2 T_{p 0}} \cong & x\left[1-\left(R_{\mathrm{NON} 1}+R_{\mathrm{NON} 2}\right) / R_{\mathrm{PSD}}\right] \\
& +x^{2}\left(R_{\mathrm{PON} 1}-R_{\mathrm{PON} 2}+R_{\mathrm{NON} 2}\right. \\
& \left.-R_{\mathrm{NON} 1}\right) / 2 R_{\mathrm{PSD}}+\left(R_{\mathrm{PON} 2}-R_{\mathrm{PON} 1}\right. \\
& \left.+R_{\mathrm{NON} 2}-R_{\mathrm{NON} 1}\right) / 2 R_{\mathrm{PSD}}
\end{aligned}
$$

Formula (5) shows that the ON resistances of the NAND gates not only cause an offset and a gain error but also the nonlinearity. Usually, the offset and gain error can easily be eliminated during the installation and calibration of the angular position sensor in its final setup. The maximum influence of $R_{\mathrm{ON}}$ occurs at $x= \pm 1$. When, for example, $R_{\mathrm{NON}}=$ $40 \Omega, \Delta R_{\mathrm{NON}}=4 \Omega, R_{\mathrm{PON}}=50 \Omega, \Delta R_{\mathrm{PON}}=5 \Omega$ and $R_{\mathrm{PSD}}=100 \mathrm{k} \Omega$, then both the maximum nonlinearity and the offset amount to $0.0045 \%$ (which corresponds to 21.1 arcsec).

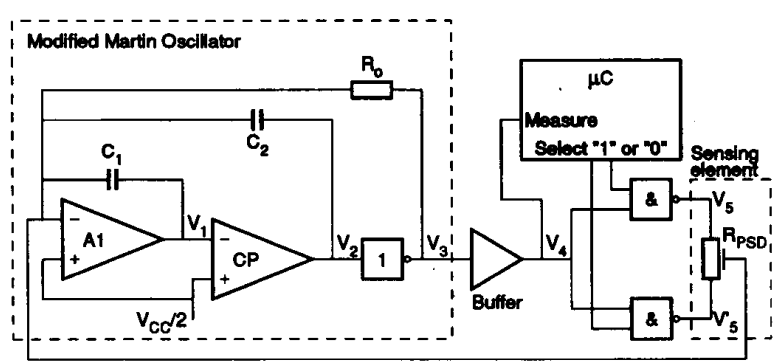

Fig. 3. The electronic circuitry of the smart resistive-capacitive position sensor system.

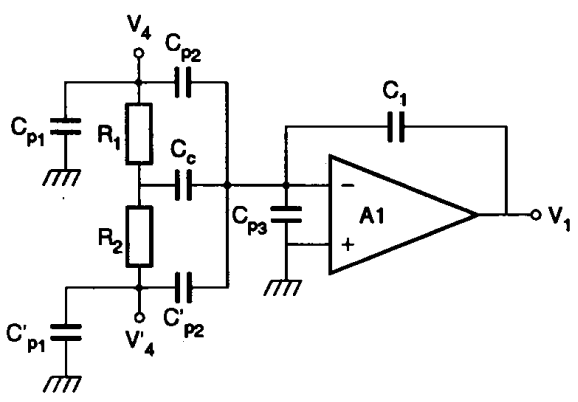

Fig. 4. The parasitic capacitances of the sensor.

\section{B. The Effect of the Parasitic Capacitors}

The parasitic capacitors of the smart angular position sensor are shown in Fig. 4.

The effects of the parasitic capacitors $C_{p 1}, C_{p 1}^{\prime}, C_{p 3}$ can be neglected, where the $C_{p 1}, C_{p 1}^{\prime}$, and $C_{p 3}$ include the cable capacitances. The parasitic capacitances $C_{p 2}$ and $C_{p 2}^{\prime}$ are also composed of two parts: the parasitic capacitances between the sliding electrode and the terminals of the resistive layer, and the parasitic capacitances between the outputs of the selector and the inverting input of the integrator in the measuring circuit.

The influence of the parasitic capacitances on the measured position can be expressed by the following equation:

$$
\frac{T_{p 1}-T_{p 2}}{T_{p 1}+T_{p 2}-T_{p 0}} \cong \frac{x+\left(C_{p 2}-C_{p 2}^{\prime}\right) / C_{c}}{1+\left(C_{p 2}+C_{p 2}^{\prime} / C_{c}\right.} .
$$

Equation (6) shows that the measured position is related to the capacitance $C_{c}$ due to the effect of the parasitic capacitances.

Since the value of capacitor $C_{c}$ is very small (about $2.2 \mathrm{pF}$ ), the parasitic capacitors $C_{p 2}$ and $C_{p 2}^{\prime}$ can cause a large error in the position measurement. This error will change with the position $x$ since the capacitor $C_{c}$ and the parasitic capacitors $C_{p 2}$ and $C_{p 2}^{\prime}$ depend on the position $x$. Therefore, the parasitic capacitances $C_{p 2}$ and $C_{p 2}^{\prime}$ have to be minimized in the design of the sensing element and measuring system.

\section{EXPERIMENT RESULTS}

The system has been built and tested using a resistivecapacitive angular sensor, a signal-processing circuit and a microcontroller of the type INTEL D87C51FA. The signalprocessing circuit uses a modified Martin oscillator [4], [5] 


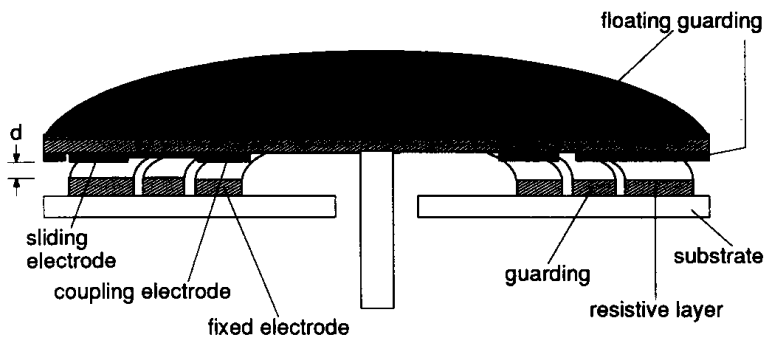

Fig. 5. The physical structure of the sensing element.

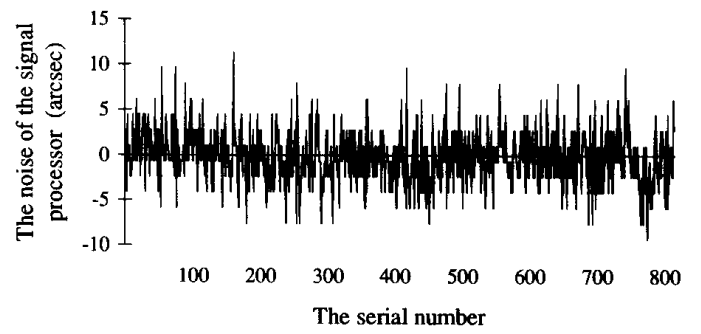

Fig. 6. The noise of the signal processor. The result of a series of 800 measurements performed under identical conditions. Each measurement takes about $100 \mathrm{~ms}$.

which has been implemented with a simple dual opamp (TLC272AC) and two CMOS NAND gates according to the circuit shown in Fig. 3. The structure of the sensing element is shown in Fig. 5. The resistive potentiometer $R_{\mathrm{PSD}}$ is about $100 \mathrm{k} \Omega$.

The electrodes were made using printed circuit board technology. The sliding electrode has an effective area of $61 \mathrm{~mm}^{2}$. The distance between the sliding electrode and the resistive layer is about $0.2 \mathrm{~mm}$, and the equivalent capacitance $C_{c}$ is about $2.2 \mathrm{pF}$. In order to eliminate the influence of the parasitic capacitors and the electromagnetic interference, a guarding electrode was made all around the sliding electrode, and the shielding is used.

The measuring system is powered by a single $5 \mathrm{~V}$ supply voltage. The frequency of the oscillator is about $7.0 \mathrm{kHz}$, and the applied range of the oscillator frequency is about 3.3 $\mathrm{kHz}-7.0 \mathrm{kHz}$. The three periods $T_{p 0}, T_{p 1}, T_{p 2}$ are measured in a total measurement time of about $100 \mathrm{~ms}$. The noise of the signal processor, which includes the sampling noise, is shown in Fig. 6.

The measured nonlinearity of the entire smart sensor system over the measurement range of $-135^{\circ}$ to $+135^{\circ}$ is less than $\pm 0.3 \%$ (see Fig. 7). This nonlinearity is mainly due to the nonlinearity of the resistive layer and the effect of the parasitic capacitors.

\section{CONCLUSION}

A novel resistive-capacitive position sensor has been presented. The signal-processing circuit is simple and can be implemented as a single-chip CMOS integrated circuit. A prototype of the system has been built and tested. The nonlinearity amounts to $0.3 \%$. The main causes of this nonlinearity are the

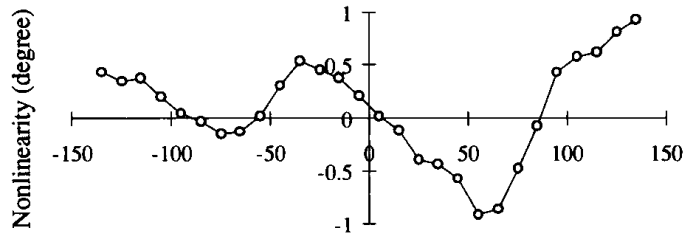

The measured angular position (degree)

Fig. 7. The experimental results for the nonlinearity of the complete smart sensor.

parasitic capacitors in the sensing element and the nonlinearity of the resistive layer.

\section{ACKNOWLEDGMENT}

The authors would like to thank G. W. de Jong, F. N. Toth, H. M. M. Kerkvliet, R. J. H. Janse, and A. F. P. van Schie for their painstaking help.

\section{REFERENCES}

[1] G. C. M. Meijer, J. van Drecht, P. C. de Jong, and H. Neuteboom, "New concepts for smart signal processors and their application to PSD displacement transducers," Sensors and Actuators, vol. A35, pp. 23-30. 1992.

[2] G. C. M. Meijer and R. Schrier, "A linear high-performance PSD displacement transducer with microcontroller interfacing," Sensors and Actuators, vol. A21-A23, pp. 538-543, 1990.

[3] Y. Z. Xing and W. J. Lian, "A novel integrated optical potentiometer," in Proc. 4th Int. Conf. Solid-State Sensors and Actuators (Transducers '87), Tokyo, Japan, June 2-5, 1987, pp. 427-430.

[4] K. Martin, "A voltage-controlled switched-capacitor relaxation oscillator," IEEE J. Solid-State Circuits, vol. SC-16, pp. 412-414, 1981.

[5] F. N. Toth and G. C. M. Meijer, "A low-cost, smart capacitive position sensor," IEEE Trans. Instrum. Meas., vol. 41, no. 6, Dec. 1992.

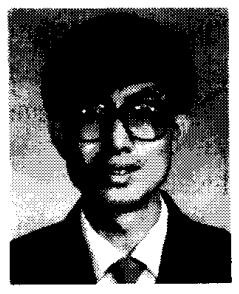

Xiujun $\mathbf{L i}$ was born in Tianjin, China, on February 19, 1963. He received the B.S. degree in physics and the M.S. degree in electrical engineering from Nankai University, Tianjin, China, in 1983 and 1986.

He joined the Department of Electronic Science, Nankai University in 1986. He is now working toward the Ph.D. degree at Delft University of Technology, Department of Electrical Engineering, The Netherlands. His research interests are in the area of the smart capacitive sensor and signal processing.

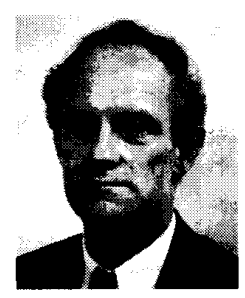

Gerard C. M. Meijer was born in Wateringen, The Netherlands, on June 28, 1945. He received the ingenieurs (M.S.) and Ph.D. degrees in electrical engineering from the Delft University of Technology, Delft, The Netherlands, in 1972 and 1982, respectively.

Since 1972 he has been part of Laboratory of Electronics, Delft University of Technology, where he is an associate professor, engaged in research and teaching on analog IC's. In 1984 and part-time during 1985-1987 he was seconded to the Delft Instruments Company in Delft, where he was involved in the development of industrial level gauges and temperature transducers.

Dr. Meijer is a member of the Netherlands Society for Radio and Electronics. 